\title{
Niveles de proteína cruda, desempeño productivo y características del huevo de gallinas de segundo ciclo
}

\section{Crude protein levels, productive performance and egg characteristics of second cycle layer hens}

\section{Eliseo Sosa-Montes 1 (D) \\ Fernando Sánchez-Morales 2 (D) \\ Arturo Pro-Martínez 3 \\ Elvia Martínez-Cruz ${ }^{1}$ (D) \\ Luis Donaldo Salinas- \\ Jiménez 1 [D, \\ Fernando González-Cerón ${ }^{1 *}$ D \\ ${ }^{1}$ Departamento de Zootecnia \\ Universidad Autónoma Chapingo. \\ CP. 56230. Texcoco, Estado de \\ México, México. \\ ${ }^{2}$ Facultad de Agronomía y \\ Veterinaria. Universidad \\ Autónoma de San Luis Potosí. \\ Soledad de Graciano Sánchez, \\ C.P 78321. S.L.P., México. \\ ${ }^{3}$ Programa de Ganadería. Cole- \\ gio de Postgraduados Campus \\ Montecillo. CP. 56230. Texcoco, \\ Estado de México, México.}

*Autor de correspondencia:

fgceron@colpos.mx

Artículo científico

Recibido: 02 de marzo 2021

Aceptado: 03 de septiembre 2021

Como citar: Sosa-Montes E, Sánchez-Morales F, Pro-Martínez A, Martínez-Cruz E, SalinasJiménez LD, González-Cerón F (2021) Niveles de proteína cruda, desempeño productivo y características del huevo de gallinas de segundo ciclo. Ecosistemas y Recursos Agropecuarios Núm. Esp. II: e2940. DOI: 10.19136/era.a8nll.2940
RESUMEN. Se evaluó el efecto de dos alimentos comerciales con diferente nivel (16 y 18\%) de proteína cruda (PC), en el desempeño productivo y las características del huevo de gallinas ponedoras de segundo ciclo. Cada tipo de alimento se asignó aleatoriamente a 210 gallinas, alojadas por pares en una jaula. La ración diaria de alimento fue $115 \mathrm{~g}$ ave ${ }^{-1}$. Durante 52 días se registraron: porcentaje de postura (PorP, \%), peso de huevo (PesH, g), masa de huevo (MasH, g ave ${ }^{-1}$ día $^{-1}$ ), conversión alimenticia (ConA, $\mathrm{g} \mathrm{g}^{-1}$ ), porcentajes de diferentes categorías de huevo y relación beneficio/costo $(\mathrm{B} / \mathrm{C})$; la producción por día fue una repetición. Los últimos ocho días del estudio se eligieron aleatoriamente 64 huevos de cada tratamiento (8 huevos $\mathrm{d}^{-1}$ ). Se registraron por huevo: longitud $(\mathrm{L}, \mathrm{cm})$, anchura $(\mathrm{A}, \mathrm{cm}$ ), índice de forma (IFH, \%), pesos de yema, clara y cascarón (PesY, PesCla y PesCas, g, respectivamente) y sus porcentajes (PorY, PorCla y PorCas, \%, respectivamente). El alimento con $18 \%$ de $P C$ produjo mejores valores $(p<0.05)$ de PorP, PesH, MasH, ConA, L y A que el alimento con $16 \%$ de PC. Las variables $\mathrm{IFH}$, PorY y $\mathrm{B} / \mathrm{C}$ no difirieron $(p>0.05)$ entre tipos de alimento. Los huevos de las aves con $18 \%$ de PC, mostraron mayor $(p<0.05)$ PorCla y menor PorCas respecto a los huevos de las aves con $16 \%$ de PC. En conclusión, el alimento comercial con $18 \%$ de PC mejoró el desempeño productivo y varias características del huevo, sin afectar la relación beneficio/costo.

Palabras clave: Desempeño productivo, gallinas ponedoras, huevo, proteína.

ABSTRACT. It was evaluated the effect of two commercial feeds with different crude protein (PC) contents (16 and $18 \%$ ) on the productive performance and egg characteristics of second-cycle hens. Each feed was randomly assigned to 210 hens that were housed two per cage. Daily ration of feed was $115 \mathrm{~g} \mathrm{bird}^{-1}$. Egg production (PorP, \%), egg weight ( $\mathrm{PesH}, \mathrm{g}$ ), egg mass (MasH g bird ${ }^{-1}$ day $^{-1}$ ), feed conversion (ConA g g ${ }^{-1}$ ), percentages of different egg categories and benefit/cost ratio $(B / C)$ were recorded during 52 days; the production of each day represented a replicate. During the last eight days, 64 eggs were randomly selected from each treatment $\left(8\right.$ eggs day $\left.^{-1}\right)$ to record in each egg: length $(L, c m)$, width $(A, c m)$, egg shape index (IFH, \%), weight of yolk, white and shell (PesY, PesCla y PesCas, respectively) and their percentages (PorY, PorCla and PorCas, \%, respectively). The $18 \%$ PC feed produced better values $(p<0.05)$ of PorP, PesH, MasH, ConA, $L$ and $A$ than the $16 \%$ PC feed. No effects $(p>0.05)$ were detected on the variables: IFH, PorY and B/C. Eggs from birds with 18\% PC showed higher $(p<0.05)$ PorCla and lower PorCas than eggs from birds with $16 \%$ PC. In conclusion, the commercial feed with 18\% PC improved productive performance and several egg characteristics, without affecting the benefit/cost ratio.

Key words: Productive performance, laying hens, egg, protein. 


\section{INTRODUCCIÓN}

El nivel de proteína cruda (PC) en el alimento, es crucial para el óptimo desempeño productivo de gallinas de postura, aceptándose ampliamente que puede mejorar la relación beneficio/costo (Laudadio et al. 2012). Se reportó que con $0.35 \%$ de metionina, al aumentar la PC de 13 a 15\%, también aumentaron $(p<0.05)$ el PorP y la MasH (Saki et al. 2015). Asimismo, se encontró que al aumentar la PC de 16 a $20 \%$, se incrementó $(p<0.05)$ el PorP y la MasH (Alagawany et al. 2011). Adicionalmente, al aumentar la PC de 15 a $16 \%$, se obtuvieron mayores $(p<0.05)$ PorP y PesH (Sohail et al. 2002). En México se usan valores entre 15 y $16 \%$ de PC en las dietas de gallinas ponedoras (Castillo et al. 2004, Carranco-Jáuregui et al. 2017, Pérez et al. 2019); bajos valores de proteína podrían afectar las características del huevo (Roberts 2004).

La muda o pelecha es un proceso que busca la renovación de los tejidos de las aves para producir huevo (Bell et al. 2004, Rafeeq et al. 2013). Las parvadas de gallinas ponedoras son inducidas a mudar, entre otras razones, para obtener producción adicional por ave durante un segundo ciclo de postura (Junqueira et al. 2006). En su segundo ciclo, las aves alcanzan 92 a 94\% de PorP respecto al primer ciclo de postura (Gjorgovska et al. 2012). Al final del segundo ciclo de postura, disminuyen el desempeño productivo y la calidad del huevo (Melo et al. 2019). Se ha hecho bastante investigación en muchos países sobre las características del huevo de primer ciclo de postura (Ramírez et al. 2016, Avellaneda et al. 2020). Sin embargo, respecto al segundo ciclo, no se conoce mucho sobre las características del huevo ni sobre el nivel de proteína de la dieta. Por tanto, el objetivo del presente estudio fue comparar dos niveles de proteína en el desempeño productivo y las características del huevo de gallinas ponedoras de segundo ciclo en México.

\section{MATERIALES Y MÉTODOS}

\section{Localidad}

El trabajo se desarrolló en la Granja Avícola Experimental del Departamento de Zootecnia, Universidad Autónoma Chapingo, Texcoco, Estado de México, México durante el año de 2019. El clima de la región es templado subhúmedo, el más seco de los subhúmedos, temperatura media anual $15.2{ }^{\circ} \mathrm{C}$, precipitación media anual de $636.5 \mathrm{~mm}$, lluvias en verano, junio a octubre (García 2004).

\section{Animales y dietas}

Dos tipos de alimento comercial con diferente nivel de proteína cruda (16 y $18 \%$ PC) se asignaron aleatoriamente a cuatrocientas veinte gallinas Bovans White de 100 semanas de edad en segundo ciclo de postura, alojadas en pares en jaulas convencionales, de manera que hubo 210 aves por tipo de alimento. La composición del alimento 16\% PC, de acuerdo a los datos de fábrica fue: mínimo $16.00 \%$ PC, mínimo $4.00 \%$ grasa cruda, máximo $5.50 \%$ fibra cruda, máximo $12.50 \%$ cenizas ( $4.2 \%$ de calcio), máximo $12.00 \%$ humedad y por diferencia $50.00 \%$ ELN. Por otro lado, la composición del alimento 18\% PC, de acuerdo a los datos de fábrica fue: mínimo $18.00 \%$ PC, mínimo $3.00 \%$ grasa cruda, máximo $5.00 \%$ fibra cruda, máximo $14.00 \%$ cenizas $(4.2 \%$ de calcio), máximo $12.00 \%$ humedad y por diferencia $48.00 \%$ ELN. El principal ingrediente proteico de los alimentos fue pasta de soya y el principal energético fue grano de sorgo. La empresa no proporcionó el aporte de aminoácidos ni de energía de los alimentos. Sin embargo, de acuerdo a la siguiente fórmula (Barzegar et al. 2019) se calculó la energía metabolizable aparente corregida al 50\% del consumo de nitrógeno (AMEs): $A M E s=3328+27.5 x E E-$ 28.8xcenizas. Dado que EE y cenizas fueron iguales, para ambas dietas (16 y 18\%) de PC, los valores de AMEs fueron $3078 \mathrm{kcal} \mathrm{kg}^{-1}$. Las dimensiones de cada jaula fueron $30 \mathrm{~cm}$ de ancho, $45 \mathrm{~cm}$ de fondo, 36 $\mathrm{cm}$ de alto en la parte posterior y $41 \mathrm{~cm}$ de alto en la parte frontal. Las jaulas estaban acopladas en módulos piramidales de dos niveles (cinco jaulas por nivel y 20 jaulas por módulo). Cada jaula contó con $30 \mathrm{~cm}$ 
de comedero de lámina y un bebedero automático de copa. Se utilizó un programa de iluminación de $16 \mathrm{~h}$ luz:8 h oscuridad. La ración diaria de alimento fue de $115 \mathrm{~g}_{\text {ave }}{ }^{-1}$ y el periodo experimental duró 52 días (d).

\section{Variables respuesta}

Las variables de desempeño productivo fueron: porcentaje de postura (PorP, \%), peso del huevo $(\mathrm{PesH}, \mathrm{g})$, masa de huevo $\left(\mathrm{MasH}, \mathrm{g}\right.$ ave ${ }^{-1}$ $\mathrm{d}^{-1}$ ) y conversión alimenticia (ConA, $\mathrm{g} \mathrm{g}^{-1}$ ). El PorP se calculó dividiendo el número de huevos entre el número de gallinas en el día de producción, y el cociente se multiplicó por 100 . El PesH se obtuvo con una báscula digital con capacidad de 200 $\mathrm{g}$ y resolución de $0.01 \mathrm{~g}$ (Modelo $\mathrm{MH}-200$, Marca MKS Tools, China). La MasH se estimó multiplicando el PorP por el PesH correspondiente. La ConA se estimó con base en el consumo de alimento (g) y la producción de huevo expresada en gramos. La relación beneficio/costo $(\mathrm{B} / \mathrm{C})$ se calculó con base en el costo de los kilogramos de alimento necesarios para producir un kilogramo de huevo, y el precio de este último. Además, se calcularon las proporciones de las siguientes categorías de huevo: primera, jumbo, chico, roto, sucio, deforme, poroso y fárfara, cuya suma produjo el $100 \%$. A fin de que los efectos de los alimentos, tuvieran tiempo suficiente para su expresión, en los últimos ocho días del periodo experimental, se colectaron aleatoriamente ocho huevos por día por tipo de alimento. Se registraron las siguientes variables en cada huevo: longitud $(\mathrm{L}, \mathrm{cm})$, anchura $(\mathrm{A}, \mathrm{cm})$, índice de forma del huevo (IFH), pesos (g) de clara, yema y cascarón (PesCla, PesY, PesCas, respectivamente), así como sus respectivos porcentajes (PorCla, PorY, PorCas). Además, se estimó el volumen $\left(\mathrm{cm}^{3}\right)$ y el área $\left(\mathrm{cm}^{2}\right)$ de cada huevo. Las variables $L$ y $A$ se determinaron con un vernier digital (Modelo HER-411, STEREN, México) con rango de medición de 0 a $150 \mathrm{~mm}$ y resolución de $0.1 \mathrm{~mm}$. El IFH se calculó a través de la expresión IFH $=\mathrm{A} / \mathrm{L}$, de acuerdo a Duman et al. (2016). Las variables PesCla, PesY, PesCas, se determinaron pesando individualmente cada porción en una báscula digital con capacidad de 200 g y resolución de $0.01 \mathrm{~g}$ (Modelo $\mathrm{MH}-200$, Marca MKS Tools, China). Posteriormente, cada valor de PesCla, PesY, PesCas se dividió entre el peso del huevo correspondiente y el cociente se multiplicó por 100 para obtener las variables PorCla, PorY y PorCas. El volumen $\left(\mathrm{cm}^{3}\right)$ y el área $\left(\mathrm{cm}^{2}\right)$ se calcularon mediante las expresiones volumen $=0.913 \times \mathrm{PesH}$ y área $=4.558 \times \mathrm{PesH}^{0.67}$, respectivamente, de acuerdo a Etches (1996); en ambos casos, el símbolo PesH correspondió al peso del huevo.

\section{Análisis estadístico}

Se realizó un análisis de varianza empleando el modelo lineal general (SAS 2011). Se consideraron dos tratamientos (alimentos con 16 y $18 \%$ de PC). Para las variables productivas, cada día del periodo experimental se consideró una repetición, es decir, cada tratamiento tuvo 52 repeticiones. Para las variables de las características del huevo, un huevo fue la unidad experimental, es decir, hubo 64 repeticiones por tratamiento.

\section{RESULTADOS}

Las aves del tratamiento con $18 \%$ de PC mostraron mejores valores ( $p<0.05$ ) de PorP, $\mathrm{PesH}$, MasH y ConA comparadas con las aves del tratamiento con $16 \%$ de PC (Tabla 1). Las aves del tratamiento con $18 \%$ de PC produjeron porcentajes mayores $(p<0.05)$ de huevos jumbo $(p<0.05)$ pero menores de huevo chico y de huevo roto $(p<0.05)$. Los demás porcentajes de huevo, incluyendo el huevo de primera fueron similares $(p>0.05)$ entre ambos tratamientos (Tabla 1).

Por otra parte, los huevos de las aves con el tratamiento $18 \%$ de PC fueron más largos, más anchos y con mayor PesCla que aquellos de las aves con el tratamiento $16 \%$ de PC $(p<0.05)$. Por tanto, los huevos provenientes del tratamiento con $18 \%$ de $P C$ tuvieron mayor volumen y área $(p<0.05)$ que aquellos del tratamiento con $16 \%$ de PC. No se encontraron diferencias $(p>0.05)$ entre tratamientos en IFH, PorY y PesCas. Sin embargo, el PorCla fue mayor mientras que el PorCas fue menor en los huevos provenientes del tratamiento con $18 \%$ de PC, 
Tabla 1. Desempeño productivo y categorías de huevo de gallinas Bovans White alimentadas con dos niveles de proteína en el alimento.

\begin{tabular}{|c|c|c|c|c|}
\hline \multirow[b]{2}{*}{ Variables productivas } & \multicolumn{2}{|c|}{ Tratamiento } & \multirow[b]{2}{*}{ EE } & \multirow[b]{2}{*}{ Valor de $p$} \\
\hline & $16 \% P C$ & $18 \% \mathrm{PC}$ & & \\
\hline PorP (\%) & $88.9^{b}$ & $90.7^{a}$ & 0.2201 & $<.0001$ \\
\hline PesH (g) & $61.5^{b}$ & $62.8^{a}$ & 0.0634 & $<.0001$ \\
\hline MasH (g) & $54.7^{b}$ & $56.9^{a}$ & 0.1565 & $<.0001$ \\
\hline ConA $\left(g g^{-1}\right)$ & $2.1^{a}$ & $2.0^{b}$ & 0.0058 & $<.0001$ \\
\hline \multicolumn{5}{|c|}{ Categorías de huevo (\%) } \\
\hline Primera & 78.5 & 77.7 & 0.3587 & 0.1125 \\
\hline Jumbo & $5.4^{b}$ & $10.9^{a}$ & 0.1735 & $<.0001$ \\
\hline Chico & $5.3^{a}$ & $1.9^{b}$ & 0.1827 & $<.0001$ \\
\hline Roto & $6.4^{a}$ & $5.4^{b}$ & 0.1901 & 0.0001 \\
\hline Sucio & 0.01 & 0.02 & 0.0084 & 0.4434 \\
\hline Deforme & 0.53 & 0.51 & 0.0574 & 0.8073 \\
\hline Poroso & 0.05 & 0.02 & 0.0170 & 0.2448 \\
\hline Fárfara & 3.9 & 3.7 & 0.1397 & 0.3328 \\
\hline
\end{tabular}

en comparación con los del otro tratamiento (Tabla 2).

\section{DISCUSIÓN}

\section{Variables productivas}

Algunos estudios no evidencian efectos en el desempeño productivo $(p>0.05)$ con dietas de 16 a $20 \%$ de PC, debido probablemente a que con $16 \%$ de $\mathrm{PC}$ se alcanzó la máxima respuesta (Junqueira et al. 2006). A niveles más bajos de proteína: al aumentar de 13 a 15\% la PC de la dieta, se observaron aumentos de $6.9 \%$ en PorP y de $7.5 \%$ en MasH (Saki et al. 2015). En este estudio, dichas variables no aumentaron tanto (2.0 y $4 \%$, respectivamente, Tabla 1$)$, debido probablemente a que los niveles de proteína de las dietas fueron altos: 16 y $18 \%$. Se reportaron resultados similares a los del presente estudio cuando se compararon dietas con 15.6 y $17 \%$ de PC, estudio en el que el PorP aumentó $(p<0.05)$ 1.5\% (Parenteau et al. 2020). Otros autores encontraron un aumento $(p<0.05)$ de $3.9 \%$ en PorP al pasar de una dieta con $16.1 \%$ de PC a otra con $17.3 \%$ de PC (Sohail et al. 2002). En el presente estudio el PorP aumentó 2.0\% (Tabla 1), valor intermedio entre 1.5 y $3.9 \%$, similarmente el valor mínimo de PC (16\%), fue intermedio entre 15.6 y $16.1 \%$.

En el estudio de Sohail et al. (2002) el PesH aumentó $1.8 \%$ y en este estudio aumentó 2.1\% (Tabla 1). Las variables PorP, PesH y MasH aumentaron linealmente $(4.0 \%, 3 \%$ y $7.3 \%)$ al aumentar la PC de 16.9 a $18.3 \%$ (De Persio et al. 2015). En este estudio, dichas variables aumentaron 2.0, 2.0 y $4.0 \%$, respectivamente (Tabla 1), en ambos casos fue mayor el aumento en $\mathrm{MasH}$, variable obtenida por multiplicación de PorP y PesH. Al aumentar la PC de 16 a $18 \%$, la variable $\mathrm{MasH}$ aumentó $12.3 \%$ (Algawani et al. 2011), en este estudio la MasH mostró un aumento menor, 4\% (Tabla 1). Esta diferencia en el cambio de la MasH se debió probablemente a la composición de las dietas. Las dietas del estudio de Algawani et al. (2011) contenían $8.0 \%$ de trigo y $1.5 \%$ de almidón de maíz, mientras que las dietas del presente estudio no contenían trigo.

El PorP y la MasH aumentaron 5.8 y $7.1 \%$, respectivamente, cuando el nivel de PC aumentó de 13.06 a $14.04 \%$ en la dieta de gallinas de 52 semanas de edad, además, esto produjo correlaciones positivas $(p<0.05)$ entre nivel de PC y PorP y entre nivel de PC y MasH (Bouyeh y Gevorgian 2011). Estos incrementos en PorP y MasH al aumentar la PC, fueron superiores a los encontrados en el presente estudio (2 y $4 \%$, respectivamente, Tabla 1). La mayor respuesta se debió a que la PC de las dietas era baja, con respecto a los requerimientos. Además, como en los estudios mencionados en el párrafo previo (De Persio 
Tabla 2. Características del huevo de gallinas Bovans White alimentadas con dos niveles de proteína en el alimento.

\begin{tabular}{|c|c|c|c|c|}
\hline \multirow{2}{*}{ Características del huevo } & \multicolumn{2}{|c|}{ Tratamiento } & \multirow{2}{*}{$\mathrm{EE}$} & \multirow{2}{*}{ Valor de $p$} \\
\hline & $16 \% P C$ & $18 \% \mathrm{PC}$ & & \\
\hline $\mathrm{L}(\mathrm{cm})$ & $5.83^{b}$ & $6.00^{a}$ & 0.0348 & 0.0009 \\
\hline$A(\mathrm{~cm})$ & $4.39^{b}$ & $4.46^{a}$ & 0.0188 & 0.0039 \\
\hline $\mathrm{IFH}$ & 0.75 & 0.75 & 0.0043 & 0.2180 \\
\hline Volumen $\left(\mathrm{cm}^{3}\right)$ & $55.98^{b}$ & $59.24^{a}$ & 0.7051 & 0.0014 \\
\hline Área $\left(\mathrm{cm}^{2}\right)$ & $71.77^{b}$ & $74.56^{a}$ & 0.6004 & 0.0013 \\
\hline PesCla (g) & $36.72^{b}$ & $39.88^{a}$ & 0.6325 & 0.0006 \\
\hline PesY (g) & $17.56^{b}$ & $18.23^{a}$ & 0.2229 & 0.0353 \\
\hline PesCas (g) & 7.08 & 6.79 & 0.1082 & 0.0602 \\
\hline PorCla (\%) & $0.60^{b}$ & $0.61^{a}$ & 0.0041 & 0.0036 \\
\hline PorY (\%) & 0.29 & 0.28 & 0.0032 & 0.1889 \\
\hline PorCas (\%) & $0.12^{a}$ & $0.10^{b}$ & 0.0016 & $<0.0001$ \\
\hline
\end{tabular}

$\overline{a, b}$ Medias con distinta literal dentro de cada fila son diferentes $(\mathrm{p}<0.05)$. IFH $=$ índice de forma del huevo, $\mathrm{L}=$ longitud del huevo, $\mathrm{A}=$ anchura del huevo, $\mathrm{PesCla}$ = peso de la clara, $\mathrm{Pes} Y=$ peso de la yema, PesCas = peso del cascarón, Por$\mathrm{Cla}=$ porcentaje de clara, PorY $=$ porcentaje de yema, PorCas $=$ porcentaje de cascarón, $\mathrm{EE}=$ error estándar de la media.

et al. 2015), el incremento de MasH fue mayor que el incremento de PorP, por ser la MasH una variable multiplicativa que involucra al PorP.

Se reportó que la ConA disminuyó linealmente $4.0 \%$ al aumentar la PC de 16.94 a $18.31 \%$ (De Persio et al. 2015), en el presente estudio la ConA también disminuyó, 3.9\% (Tabla 1). Contrariamente, Parenteau et al. (2020) reportaron un aumento de $4.1 \%$ en la ConA cuando la PC aumentó de 15 a 17\%.

Con las dietas del estudio de Parenteau et al. (2020), el porcentaje de huevo jumbo aumentó $77 \%$ $(p<0.05)$ al aumentar la PC de 14 a $16 \%$, y no cambiaron las demás categorías de huevo. En este estudio, al incrementarse la PC de la dieta, el aumento en huevo jumbo fue de $103.5 \%$ (Tabla 1, p < 0.05). En el presente estudio, al aumentar la PC de la dieta, los porcentajes de huevo chico y roto disminuyeron $(p<$ $0.05)$, y no cambiaron los porcentajes de las demás categorías de huevo $(p>0.05)$.

\section{Características del huevo}

El PorCla aumentó 1.8\% $(p<0.05)$ al aumentar la PC de 16.1 a 17.4\% (Wu et al. 2007) y en este estudio el PorCla aumentó 2.9\% (Tabla 2). Moula et al. (2010) reportaron en gallina ponedora comercial, los siguientes valores de porcentajes de clara, yema y cascarón: PorCla (59.4\%), PorY (28.6\%) y PorCas $(12.0 \%)$ muy parecidos a los del presente experimento con $16 \%$ de PC (Tabla 2). Al aumentar el nivel de PC de 14.89 a $17.38 \%$ en la dieta, se encontró que el PorCas disminuyó ( $p$ < 0.05) 4.3\% (Gunawardana et al. 2008). En este estudio el PorCas también disminuyó $10.5 \%(p<0.05$, Tabla 2). Esta disminución del PorCas se debe a que el PesCla y el PesY aumentaron ( $p<0.05$, Tabla 2$)$, mas no lo hizo el PesCas ( $P>0.05)$; por tanto, el huevo adquiere más clara y más yema al aumentar la PC de la dieta, pero puede comprometerse la resistencia del cascarón, ya que el peso del cascarón no cambia y sí disminuye su proporción (Tabla 2). Estos incrementos de los pesos de la clara y de la yema son muy importantes para el consumidor, pero son aún más importantes para los desarrollos embrionario y postnatal del ave (Willems et al. 2014).

Las variables largo y ancho del huevo (Tabla 2) fueron similares a las reportadas en la literatura: 5.3 y $4.0 \mathrm{~cm}$, respectivamente (Ukwu et al. 2017), 5.6 y $4.4 \mathrm{~cm}$, respectivamente (Moula et al. 2010). Estas variables se incorporan en la variable IFH (Adeyemo et al. 2012). En este trabajo, las variables largo y ancho del huevo aumentaron $(p<0.05) 2.9$ y $1.8 \%$, respectivamente al aumentar el nivel de PC de la dieta. Por tanto, el IFH no cambió ( $p>0.05$ ) al aumentar la PC de la dieta, debido a que el largo y ancho del huevo cambiaron en forma directamente proporcional (Tabla 2). El IFH no cambió $(P>0.05)$ cuando se 
estimó en el huevo de diferentes cruzas de gallinas ponedoras (Prakash et al. 2020). El IFH varió de 76.3 a $78.3 \%$ entre gallinas comerciales (Moula et al. 2010), valores levemente superiores a los obtenidos en el presente estudio (Tabla 2). Además, al aumentar las variables longitud y ancho del huevo, se incrementaron $(p<0.05)$ el área y el volumen: 3.9 y $5.8 \%$, respectivamente (Tabla 2 ); es decir, el huevo resultó más grande al aumentar la PC de la dieta.

\section{Relación beneficio/costo}

Alagawany et al. (2011) reportaron que la eficiencia económica fue mejor al pasar la PC de la dieta, de 16 a $18 \%$, pero disminuyó cuando la PC de la dieta fue $20 \%$. En este trabajo las relaciones $\mathrm{B} / \mathrm{C}$ fueron: $1.45 \pm 0.04$ y $1.55 \pm 0.04$, para las dietas con 16 y $18 \%$ PC, respectivamente, pero no fueron estadísticamente diferentes $(p>0.05)$. Lacadin y Humag (2016) obtuvieron una $\mathrm{B} / \mathrm{C}$ de 1.9 usando una dieta maíz-pasta de soya con $17 \%$ de $\mathrm{PC}$, relación mayor que las obtenidas en el presente trabajo. Winkler et al. (2017) no encontraron efectos económicos entre una dieta control con $14.74 \%$ de PC y una dieta con $15.58 \%$ de PC, entre otras dietas. Probablemente la razón por la cual no se observan efectos económicos al aumentar la PC de la dieta es que el costo de los ingredientes proteicos es más alto que el de los ingredientes energéticos, y aunque aumente PorP, mejore la ConA y se produzcan huevos más grandes, el ingreso no supera al mayor costo de la dieta (De Persio et al. 2015). Además, en algunas estimaciones económicas, las ganancias se calculan por docena, el huevo tiende a ser más grande que el proveniente de las dietas con menos PC, y los precios de ingredientes y de huevo son variables (Winkler et al. 2017; Gunawardana et al. 2008).

\section{CONCLUSIONES}

Aumentar la proteína de 16 a $18 \%$ en la dieta para gallinas ponedoras de segundo ciclo, mejora el desempeño productivo y algunas características del huevo, tales como peso de clara, peso de yema y porcentaje de clara, disminuye el porcentaje de cascarón y no afecta la relación beneficio/costo.

\section{LITERATURA CITADA}

Alagawany M, El-Hindawy MM, Ali AA, Soliman MM (2011) Protein and total sulfur amino acids relationship effect on performance and some blood parameters of laying hens. Egyptian Journal of Nutrition and Feeds 14: 477-487.

Adeyemo GO, Abioye SA, Aderemi FA (2012) The effect of varied dietary crude protein levels with balanced amino acids on performance and egg quality characteristics of layers at first laying phase. Food and Nutrition Sciences 3: 526-529.

Avellaneda Y, Ariza-Nieto C, Afanador-Téllez G (2020) Crude glycerin and energy density of diets for growing, pre-lay and pre-peak Backcob Brown egg-laying hens. Brazilian Journal of Poultry Science 22(02). DOI: 10.1590/1806-9061-2019-1179.

Barzegar S, Wu SB, Noblet J, Choct M, Swick RA (2019) Energy efficiency and net energy prediction of feed in laying hens. Poultry Science 98: 5746-5758.

Bell D, Chase B, Douglass A, Hester P, Mench J, Newberry R, Shea-Moore M, Stanker L, Swanson J, Armstrong $J(2004)$ UEP uses scientific approach in its establishment of welfare guidelines. Feedstuffs 76: 1-10.

Bouyeh M, Gevorgian OX (2011) Influence of different levels of lysine, methionine and protein on the performance of laying hens after peak. Journal of Animal and Veterinary Advances 10: 532-537.

Carranco-Jáuregui ME, Carrillo-Domínguez S, Fuente-Martínez B, Ávila-González E, Solano ML (2017) Cambios de la fracción hidrosoluble de huevo de gallinas alimentadas con harina de camarón almacenado a 
diferentes tiempos y temperaturas. Revista Mexicana de Ciencias Pecuarias 8: 365-373.

Castillo C, Cuca M, Pro A, González M, Morales E (2004) Biological and economic optimum level of calcium in white Leghorn laying hens. Poultry Science 83: 868-872.

De Persio S, Utterback PL, Utterback CW, Rochell SJ, O'Sullivan N, Bregendahl K, Arango J, Parsons CM, Koelkebeck KW (2015) Effects of feeding diets varying in energy and nutrient density to Hy-Line W-36 laying hens on production performance and economics. Poultry Science 94: 195-206.

Duman M, Şekeroğlu A, Yildirim A, Eleroğlu H, Camci Ö (2016) Relation between egg shape index and egg quality characteristics. European Poultry Science 80: 1-9.

Etches RJ (1996) Reproducción aviar. Acribia. Zaragoza, España. 339p.

García E (2004) Modificaciones al sistema de clasificación climática de Köppen. Instituto de Geografía-UNAM. México. 90P.

Gjorgovska N, Filev K, Levkov V, Kostadinov T, Jusufi E (2012) Egg production during the artificial moulting and a new laying cycle of aged hens. Archiva Zootechnica 15: 53-60.

Gunawardana P, Roland DA, Bryant MM (2008) Effect of energy and protein on performance, egg components, egg solids, egg quality, and profits in molted Hy-Line W-36 hens. Journal of Applied Poultry Research 17: 432-439.

Junqueira OM, Laurentiz AC, Silva FR, Rodriguez EA, Casartelli EM (2006) Effects of energy and protein levels on egg quality and performance of laying hens at early second production cycle. Journal of Applied Poultry Research 15: 110-115.

Lacadin AL, Humag JJP (2016) Cattle stomach contents concentrate as supplemental lysine and methionine source for single combed white leghorn layers on plant protein-based diets. International Journal of Science and Research 5: 695-698.

Laudadio V, Passantino L, Perillo A, Lopresti G, Passantino A, Khan UR, Tufarelli V (2012) Productive performance and histological features of intestinal mucosa of broiler chickens fed different dietary protein levels. Poultry Science 91: 265-270.

Melo RD, Cruz FGG, Rufino JPF, Melo LD, Feijo JC, Oliveira Filho PA (2019) Performance and egg quality of laying hens evaluated in different periods postmolt. Revista Científica de Avicultura e Suinocultura 5: 54-62.

Moula N, Moussiaux AN, Decuypere E, Farnir F, Mertens K, Baerdemaeker J, Leroy P (2010) Comparative study of egg quality traits in two Belgian local breeds and two comercial lines of chickens. Archiv fur Geflugelkunde 74: 164-171.

Parenteau Al, Stevenson M, Kiarie GE (2020) Egg production and quality responses to increasing isoleucine supplementation in shaver white hens fed a low crude protein corn-soybean meal diet fortified with synthetic amino acids between 20 and 46 weeks of age. Poultry Science 99: 1444-1453.

Pérez MJ, Cuca G JM, Ramírez VG, Carrillo DS, Pro MA, Ávila GE, Sosa ME (2019) Evaluation of two soybean soapstock in egg production and quality in Bovans hens. Revista Mexicana de Ciencias Pecuarias 10: 283-297.

Prakash J, Vipin, Kumar K, Pandey Y, Khumar KR (2020) Egg production and Egg Quality Characteristics in Direct and Reciprocal Crosses using CARI Nirbheek and CARI Shyama. Journal of Animal Research 10: 579-683. 
Rafeeq M, Rashid N, Awan MA, Tariq MM, Abbas F, Ahmed Z, Taj I (2013) Effect of forced molting on body characteristics and post-molting egg production performance of layers in Quetta, Pakistan. Brazilian Journal of Poultry Science 15: 347-352.

Ramírez A, González J, Andrade V, Torres V (2016) Efecto de los tiempos de conservación a temperatura ambiente, en la calidad del huevo de gallinas camperas (Gallus domesticus) en la Amazonia Ecuatoriana. Revista Electrónica de Veterinaria 17: 1-17.

Roberts RJ (2004) Factors affecting egg internal quality and egg shell quality in laying hens. Journal of Poultry Science 41: 161-177.

Saki AA, Zamani P, Haghi M, Aliarabi H, Malecky M, Rahmatnejad E, Mirzale S (2015) Molecular aspects, blod parameters, bone mechanical properties, and performance of laying hens in response to various levels of methionine and protein. Revue de Medecine Veterinaire 166: 47-53.

SAS (2011). Base SAS ${ }^{\circledR}$ 9.3 Procedures Guide. USA: SAS Institute. Cary, NC, USA. 528p.

Sohail SS, Bryant MM, Voitle AR, Roland AD (2002) Influence of calsporin on commercial Leghorns. Journal of Applied Poultry Research 11: 379-387.

Ukwu HO, Ezihe CO, Assa SK, Anyogo ME (2017) Effect of egg weight on external and internal egg quality traits of Isa Brown egg layer chickens in Nigeria. Journal of Animal Science and Veterinary Medicine 2: 126-132.

Willems E, Decuypere E, Buyse J, Everaert N (2014) Importance of albumen during embryonic development in avian species, with emphasis on domestic chicken. World's Poultry Science Journal 70: 503-518.

Winkler RL, Murphy MK, Hermes CJ (2017) Three hulles oat varieties show economic potential as organic layer feed grain. Renewable Agriculture and Food Systems 35: 418-431.

Wu G, Gunawardana P, Bryant MM, Roland AD (2007) Effect of dietary energy and protein on performance, egg composition, egg solids, egg quality and profits of Hy-line W-36 hens during phase 2. International Journal of Poultry Science 6: 739-744. 